\title{
Positron Emission Tomography Imaging of Endometrial Cancer Using Engineered Anti-EMP2 Antibody Fragments
}

\author{
Maoyong Fu, ${ }^{1}$ Sarah Brewer, ${ }^{1}$ Tove Olafsen, ${ }^{2}$ Anna M. Wu, ${ }^{2,3}$ Lynn K. Gordon, ${ }^{4,5}$ \\ Jonathan Said, ${ }^{1,3}$ Jonathan Braun, ${ }^{1,2,3}$ Madhuri Wadehra ${ }^{1,3}$ \\ ${ }^{1}$ Department of Pathology and Laboratory Medicine, David Geffen School of Medicine at UCLA, Los Angeles, CA 90095, USA \\ ${ }^{2}$ Department of Molecular and Medical Pharmacology, David Geffen School of Medicine at UCLA, Los Angeles, CA 90095, USA \\ ${ }^{3}$ Jonsson Comprehensive Cancer Center, David Geffen School of Medicine at UCLA, Los Angeles, CA 90095, USA \\ ${ }^{4}$ Department of Ophthalmology, Jules Stein Eye Institute, David Geffen School of Medicine at UCLA, Los Angeles, CA 90095, USA \\ ${ }^{5}$ Department of Surgery, Greater Los Angeles Veterans Affairs Healthcare System, Los Angeles, CA 90099, USA
}

\begin{abstract}
Purpose: As imaging of the cell surface tetraspan protein epithelial membrane protein-2 (EMP2) expression in malignant tumors may provide important prognostic and predictive diagnostic information, the goal of this study is to determine if antibody fragments to EMP2 may be useful for imaging EMP2 positive tumors.

Procedures: The normal tissue distribution of EMP2 protein expression was evaluated by immunohistochemistry and found to be discretely expressed in both mouse and human tissues. To detect EMP2 in tumors, a recombinant human anti-EMP2 minibody (scFv-hinge- $\mathrm{C}_{\mathrm{H}} 3$ dimer; $80 \mathrm{kDa}$ ) was designed to recognize a common epitope in mice and humans and characterized. In human tumor cell lines, the antibody binding induced EMP2 internalization and degradation, prompting the need for a residualizing imaging strategy. Following conjugation to DOTA $\left(1,4,7,10\right.$-tetraazacyclododecane- $N, N^{\prime}, N^{\prime}, N^{\prime \prime}$-tetraacetic acid), the minibody was radiolabeled with ${ }^{64} \mathrm{Cu}\left(t_{1 / 2}=12.7 \mathrm{~h}\right)$ and evaluated in mice as a positron emission tomography (PET) imaging agent for human EMP2-expressing endometrial tumor xenografts.

Results: The residualizing agent, ${ }^{64} \mathrm{Cu}$-DOTA anti-EMP2 minibody, achieved high uptake in endometrial cancer xenografts overexpressing EMP2 (10.2 \pm 2.6 , percent injected dose per gram $(\% \mathrm{ID} / \mathrm{g}) \pm \mathrm{SD})$ with moderate uptake in wild-type HEC1A tumors $(6.0 \pm 0.1)$. In both cases, precise tumor delineation was observed from the PET images. In contrast, low uptake was observed with anti-EMP2 minibodies in EMP2-negative tumors (1.9 \pm 0.5$)$.

Conclusions: This new immune-PET agent may be useful for preclinical assessment of antiEMP2 targeting in vivo. It may also have value for imaging of tumor localization and therapeutic response in patients with EMP2-positive malignancies.
\end{abstract}

Key words: Epithelial membrane protein-2, Endometrial cancer, PET imaging, Minibody, Copper-64

Correspondence to: Madhuri Wadehra; e-mail: mwadehra@mednet. ucla.edu

\section{Introduction}

promising avenue in molecular imaging is the development
of agents that detect the presence and levels of molecular
biomarkers in malignant tumors. In this way, molecular 
imaging may provide important information for patient stratification to targeted therapies, aid in restaging diseases, and be used to monitor treatment response. Thus, new biomarkers that may aid in patient management are urgently needed.

Epithelial membrane protein-2 (EMP2) is a novel oncogene upregulated in a number of gynecological tumors. A member of the growth arrest specific-3/peripheral myelin protein-22 (GAS3/PMP22) family of tetraspan proteins, overexpression of EMP2 is associated with tumor progression as well as poor patient survival $[1,2]$. Its expression is detected in up to $60 \%$ of endometrial tumors and $75 \%$ of advanced ovarian tumors [1-3].

Functionally, EMP2 appears to be important for trafficking, intracellular compartmentalization, and the surface display of selected receptors and glycolipids [4]. EMP2 physically associates with and regulates the activity of integrin-FAK signaling complexes, with the consequence that high levels of EMP2 increase tumor cell invasion [5-8]. These findings, concordant with the role of other members of the tetraspan family, indicate that EMP2 organizes molecules at the cell surface that are brought into engagement with specific signaling complexes, therefore controlling downstream biological responses [9-12].

We have previously reported an anti-EMP2 diabody (scFv dimer; $55 \mathrm{kDa}$ ) derived from a human immunoglobulin V-gene phage display library that recognizes a shared epitope on murine and human EMP2 [13]. Localized treatment with the anti-EMP2 diabody showed therapeutic efficacy with reduced tumor load in xenograft models for endometrial and ovarian tumors. Given that EMP2 overexpression plays an important role in aggressive tumor behavior and poor clinical outcome [1], early stage detection and quantification of EMP2 may be clinically relevant and used for selection of optimal therapy for individual patients.

Positron emission tomography (PET) imaging is a widely used imaging modality due to its higher image resolution and sensitivity compared to single-photon imaging [14]. In oncology, PET most commonly employs 2-deoxy-2- $\left[{ }^{18} \mathrm{~F}\right]$ fluoro-D-glucose $\left(\left[{ }^{18} \mathrm{~F}\right] \mathrm{FDG}\right)$ as a tracer for imaging elevated metabolism in tumors [15]. However, $\left[{ }^{18} \mathrm{~F}\right]-\mathrm{FDG}$ has certain limitations in tumor detection. First, it is not specific for tumor detection, as inflammation and wound healing also exhibit tissue uptake of $\left[{ }^{18} \mathrm{~F}\right]-\mathrm{FDG}[16]$. Second, with regard to gynecological tumors, $\left[{ }^{18} \mathrm{~F}\right]$-FDG uptake is deficient in a substantial fraction of gynecologic malignancies and can be insensitive for assessing responses to certain antitumor therapies [17-19].

Here, we have generated a larger anti-EMP2 antibody fragment, a minibody (scFv-hinge- $\mathrm{C}_{\mathrm{H}} 3$ dimer; $\left.80 \mathrm{kDa}\right)$. In order to determine if EMP2 could be detected in vivo, the minibody was conjugated to DOTA and radiometal labeled with the positron emitter ${ }^{64} \mathrm{Cu}\left(\mathrm{T}_{1 / 2}=12.7 \mathrm{~h}\right)$. Small animal PET imaging showed excellent tumor targeting of the ${ }^{64} \mathrm{Cu}$ DOTA-minibody in mice bearing EMP2 positive endometrial tumor xenografts. Thus, whole-body PET detection of this anti-EMP2 antibody fragment may have promise as a potential imaging agent for staging and monitoring of EMP2-positive endometrial tumors.

\section{Materials and Methods}

\section{Cell Lines and Cell Culture}

HEC1A/EMP2 cells were generated by stable transfection with the EGFP-N3 expression vector bearing human EMP2 as previously described [7]. Ramos cells were purchased from American Type Culture Collection (ATCC, Manassas, VA, USA), and the murine mammary tumor D2F2 was kindly provided by Dr. Manuel Penichet (UCLA). The cells were cultivated at $37{ }^{\circ} \mathrm{C}$ in a humidified $5 \% \mathrm{CO}_{2}$ in DMEM medium (Mediatech, Manassas, VA, USA) supplemented with $10 \%$ fetal calf serum (Hyclone Laboratories, Logan, UT, USA), 2 mM L-glutamine, $1 \mathrm{mM}$ sodium pyruvate, $100 \mathrm{U} / \mathrm{ml}$ penicillin, and $100 \mathrm{U} / \mathrm{ml}$ streptomycin (all from Invitrogen Life Technologies, Carlsbad, CA, USA).

\section{Institutional Review}

All procedures with and tissue procurement from mice were performed under Institutional Review Board (IRB) approval. All human samples were obtained from the UCLA Department of Pathology and Laboratory Medicine under Institutional Review Board exemption approval.

\section{Construction and Production of Anti-EMP2 Antibody Fragments}

The anti-EMP2 minibody was generated using published methods [20]. Briefly, genes encoding the variable KS83 domain were assembled by PCR into a single-chain Fv fragments (scFv) [13] and cloned into pCR2.1-TOPO vector (Invitrogen). The genes encoding the human IgG1 hinge and $\mathrm{C}_{\mathrm{H}} 3$ domain (hinge- $\mathrm{C}_{\mathrm{H}} 3$ ) were also assembled by PCR and cloned into another pCR2.1-TOPO vector. The pCR2.1-TOPO-scFv and scFv-hinge- $\mathrm{C}_{\mathrm{H}} 3$ were digested and inserted into the pcDNA3.1myc/his(-) mammalian expression vector (Invitrogen) at $X b a \mathrm{I}$ and EcoRI site and XhoI and EcoRI, respectively. The resultant anti-EMP2 minibody was termed KS83.

Chinese hamster cells (CHO-K1) were transfected with $30 \mu \mathrm{g}$ of linearized pcDNA3.1-KS83 vector DNA and selected using $1 \mathrm{mg} / \mathrm{ml}$ of geneticin (G418, Sigma-Aldrich, St. Louis, MO, USA) as described previously [21]. After approximately 2 weeks, cells were screened for expression by enzyme-linked immunosorbent assay (ELISA) using goat anti-human Fc-specific antibody (Jackson Immunoresearch, West Grove, PA, USA). The highest producers were identified, and the cells were subcloned through serial dilutions to isolate individual clones. Cells from high expressing individual clones were expanded in Celline AD 1000 bioreactor flasks (IBS Integra Biosciences, Hudson, NH, USA) according to the manufacturer's instructions. The KS 83 minibody was purified from supernatant by Protein-L affinity chromatography (Thermo Fisher Scientific Inc., Rockford, IL, USA), using phosphatebuffered saline (PBS) as running buffer. The elution buffer was using $30 \% 0.2 \mathrm{M}$ citrate buffer $(\mathrm{pH} 2.1)$ in PBS into $80 \% v / v$ 
1 M Tris base, $\mathrm{pH}$ 8.2. Purified minibody was dialyzed against PBS and concentrated using Amicon Ultra-4 (Millipore, Billerica, MA, USA). The final concentration of purified minibodies was determined by a Nanodrop 2000 (Thermo Scientific).

\section{Enzyme-linked immunosorbent assay}

The anti-EMP2 antibody fragments were captured by the peptides corresponding to the extracellular loop of human EMP2 [13], and ELISA was performed as described previously [13]. Specifically, bound minibodies were detected with horseradish peroxidase (HRP)-conjugated goat antihuman Fc-specific antibody (Jackson Immunoresearch), followed by tetramethylbenzidine solution (eBioscience, San Diego, CA, USA). Plates were read using a Model 550 microplate reader (Bio-Rad, Hercules, CA, USA) at $450 \mathrm{~nm}$.

\section{Flow Cytometry}

HEC1A/EMP2 cells $\left(1 \times 10^{6}\right)$, murine D2F2 cells, or Ramos cells suspended in $1 \mathrm{ml}$ of flow buffer (PBS, $0.2 \%$ bovine serum albumin, and $0.02 \%$ sodium azide), were centrifuged for $5 \mathrm{~min}$ at $500 \times g, 4{ }^{\circ} \mathrm{C}$. Cells were resuspended in $0.1 \mathrm{ml}$ flow buffer and incubated with $0.5 \mu \mathrm{g}$ of the primary antibody for $1 \mathrm{~h}$ at $4{ }^{\circ} \mathrm{C}$ on a rotator. Cells were washed three times with flow buffer and incubated for $30 \mathrm{~min}$ at $4{ }^{\circ} \mathrm{C}$ with phycoerythrin (PE)-conjugated goat antihuman Fc-specific antibody (Jackson Immunoresearch) in $0.1 \mathrm{ml}$ flow buffer. Following three washes, cells were resuspended in $0.4 \mathrm{ml}$ flow buffer, and flow cytometry was immediately performed. Fc receptors on Ramos cells were blocked by incubation for $15 \mathrm{~min}$ with $50 \mu \mathrm{l} \mathrm{Fc}$ block solution (heat inactivated rabbit normal serum, 1:10 dilute in PBS, freeze-thaw five cycles).

\section{Immunohistochemistry}

Mouse and human tissues were stained for EMP2 expression as previously described [22]. Normal human tissue were stained using an array created by the immunohistochemistry core of the Translational Pathology Core Laboratory. This array contains 248 spots from 30 tissues with examples of every type from at least two patients, and all samples were verified as being normal by histology. All positive staining was verified independently using additional full tissue sections from two additional patients. For mouse tissue, organs were extracted, tissue fixed in $10 \%$ formalin, embedded in paraffin, and sectioned at $5-\mu \mathrm{m}$ thickness. To assess EMP2 expression in all tissue, antigen retrieval was performed using $0.1 \mathrm{M}$ citrate buffer $(\mathrm{pH} 6.0)$ at $95{ }^{\circ} \mathrm{C}$ for $20 \mathrm{~min}$. The slides were incubated with rabbit antimouse EMP2 antisera (1:250) [23], antihuman EMP2 antisera (1:800) [1] or control rabbit serum at the same dilutions overnight. The antibody signal was visualized using the Vectastain ABC kit (Vector Labs, Burlingame, CA, USA) according to the manufacturer's instructions. EMP2 expression was detected using diaminobenzidine, and nuclei were counterstained using hematoxylin. Semiquantitative scoring of the EMP2-stained tissue was conducted using a $0-3$ intensity scale.

\section{Western Blot Analysis}

Mouse tissues were processed using fresh specimens and immediately frozen on dry ice. Frozen human tissue samples of postdiagnostic remnant surgical resection specimens obtained from the UCLA Department of Pathology and Laboratory Medicine. Tissues were homogenized using cold radioimmunoprecipitation assay buffer and then resuspended in Laemmli buffer. To detect EMP2 expression, cell extracts were treated with peptide $\mathrm{N}$ glycosidase F (New England Biolabs, Beverly, MA, USA) to deglycosylate the proteins. The lysates were separated on a $12 \%$ sodium dodecyl sulfate polyacrylamide gel electrophoresis (SDSPAGE) gel, and proteins were transferred onto nitrocellulose (BioRad Laboratories, Hercules, CA, USA). EMP2 was detected using rabbit antihuman EMP2 (1:2000) antiserum followed by a HRPconjugated goat antirabbit $\operatorname{IgG}$ and enhanced chemiluminescence detection reagents (GE Healthcare, Piscataway, NJ, USA).

\section{Immunofluorescence}

HEC1A/EMP2 cells were plated onto glass coverslips (Fisher Scientific, Pittsburgh, PA, USA). Cells were washed in PBS and then incubated at various time points with $20 \mu \mathrm{g} / \mathrm{ml}$ of the control A10 or the anti-EMP2 diabody. To visualize the diabody, cells were fixed in $1.6 \%$ formaldehyde for $20 \mathrm{~min}$ followed by incubation with a fluorescein isothiocyanate-conjugated anti-Myc antibody (Invitrogen). To visualize EMP2 staining, cells were fixed and permeabilized in methanol at $-20{ }^{\circ} \mathrm{C}$ for $30 \mathrm{~min}$ and rehydrated in PBS. Cells were blocked in $1 \%$ normal goat serum for $45 \mathrm{~min}$ and incubated overnight at $4{ }^{\circ} \mathrm{C}$ with rabbit antihuman EMP2 antisera $(1: 400)$ in a humidified chamber. Cells were rinsed with PBS $/ 0.01 \%$ Triton X-100 and then incubated $(2-4 \mathrm{~h}$ at room temperature) with rhodamine-conjugated goat antirabbit IgG (1:4,000; Jackson Immunoresearch). Negative controls included incubation of cells with secondary antibody alone. Cells were copiously washed in PBS $/ 0.01 \%$ Triton X-100, rinsed briefly with double deionized $\mathrm{H}_{2} \mathrm{O}$, and mounted in Vectamount HardSet with 4',6-diamidino-2-phenylindole solution. Images were taken using an Olympus BX51 light microscope coupled with the DP72 digital camera at $400 \times$ magnification.

\section{DOTA Conjugation and Radiolabeling with ${ }^{64} \mathrm{Cu}$}

The KS83 minibody was conjugated with 1,4,7,10-tetraazacyclododecane $N, N^{\prime}, N^{\prime \prime}, N^{\prime \prime \prime}$-tetraacetic acid mono- $N$-hydroxysuccinimide ester (DOTA-NHS-ester; Macrocyclics, Dallas, TX, USA) according to the method described previously [24]. Different conjugation molar ratios of DOTA to minibody $(20,50,100$, and 1,000) were used in order to determine the condition that would preserve the binding activity of the conjugate. ${ }^{64} \mathrm{Cu}$ (copper chloride in $0.1 \mathrm{M}$ $\mathrm{HCl}$; radionuclide purity, $>99 \%$ ) was provided by the Mallinckrodt Institute of Radiology (Washington University School of Medicine). For radiometal labeling, $200 \mu \mathrm{g}$ of DOTA-conjugated KS83 was incubated with $400 \mu \mathrm{Ci}$ (14.8 MBq) of ${ }^{64} \mathrm{Cu}$ in $0.1 \mathrm{M} \mathrm{NH}_{4}$ citrate $(\mathrm{pH}$ 5.5) for $50 \mathrm{~min}$ at $43{ }^{\circ} \mathrm{C}$. The radiolabeling efficiency was determined using instant thin-layer chromatography with Tec-Control Chromatography strips according to the manufacturer's instruction 
(Biodex Medical Systems, Shirley, NY, USA). In some experiments, the anti-CD20 minibody was used as a negative control isotype tracer [25]. DOTA conjugation and radiolabeling with ${ }^{64} \mathrm{Cu}$ were performed in the same way as above.

\section{Immunoreactivity of Radiolabeled Minibody}

Immunoreactivity was measured as previously described [21]. In this study, HEC1A/EMP2 cells $\left(6-9 \times 10^{7}\right)$ in excess were incubated with $20 \mathrm{ng}$ of ${ }^{64} \mathrm{Cu}$-DOTA-KS83 for $1 \mathrm{~h}$ on ice. Cells were centrifuged at $1,000 \times g$ for $5 \mathrm{~min}$, and the activity remaining in the supernatant was counted using a Wallac WIZARD automatic $\gamma$-counter (Perkin-Elmer Life and Analytic Sciences Inc., Waltham, MA, USA). The immunoreactivity (\%IR) of the radiolabeled antibody was calculated with the following formula: $\% \mathrm{IR}=100-($ activity from the tubes with cells activity from control tubes without cells $\times 100$ ).

\section{Tumor Xenograft Murine Model}

All procedures involving animals were performed under approved protocols of the UCLA Animal Research Committee. Six to 8-week-old female BALB/c nude mice (Charles River Labs, Wilmington, MA, USA) were injected subcutaneously in the shoulder with $1 \times 10^{5}$ of HEC1A/EMP2 or wildtype HEC1A cells 3-4 weeks prior to imaging. $5 \times 10^{6}$ Ramos cells were injected to form the subcutaneous tumor $1-2$ weeks prior to imaging. Each group utilized five mice per experiment.

\section{Small-Animal PET Imaging}

Mice were anesthetized with $2 \%$ isoflurane prior to intravenous injection of $\sim 100 \mu \mathrm{Ci}(3.7 \mathrm{MBq})$ of ${ }^{64} \mathrm{Cu}$-DOTA-KS 83 minibody $(50 \mu \mathrm{g})$ or ${ }^{64} \mathrm{Cu}-\mathrm{DOTA}-\mathrm{CD} 20$ minibody $(50 \mu \mathrm{g})$ via tail vein (specific activity, $0.074 \mathrm{MBq} / \mu \mathrm{g}$ ). Mice were imaged at 4 and $20 \mathrm{~h}$ post-injection. A cylinder of known weight, containing a known amount of radioactivity, was scanned to provide a calibration standard. Mice were serially imaged using the micro-PET Focus 220 PET Scanner (Siemens Preclinical Solutions, Knoxville, TN, USA). To enable imaging, mice were positioned in a prone position along the long axis of the microPET scanner and imaged. Images were reconstructed using a filtered backprojection reconstruction algorithm [26]. Directly after the final microPET scan, a CT scan was performed using a MicroCAT II Scanner (Concorde Microsystems, Knoxville, TN, USA).

\section{Biodistribution and ROI Analysis}

After the final imaging scan, the mice were euthanized, major organs and tumors were removed and weighed, and radioactivity was counted using a Wallac WIZARD automatic $\gamma$-counter (PerkinElmer Life and Analytic Sciences Inc.). The percent injected dose per gram of tissue $(\% \mathrm{ID} / \mathrm{g})$ was calculated to represent the radioactivity uptake in tumor and organs and decay corrected.
AMIDE was used to analyze overlaid CT and PET scans [27]. Regions of interest (ROIs) were drawn based on the CT image. ROI statistics were generated using AMIDE and converted to percent injected dose per gram after input of the individual decay corrected dose and cylinder calibration factor.

\section{Statistical Analysis}

All significant differences between groups were evaluated using two-tailed Student's unpaired $t$ test or ANOVA at a $95 \%$ confidence level (GraphPad Prism version 3.0; GraphPad Software, La Jolla, CA, USA), and $P<0.05$ were considered significant.

\section{Results}

\section{Expression of the EMP2 Protein}

To determine the suitability of EMP2 as a new molecular imaging target, its tissue expression in mouse and human was evaluated (Fig. 1). Western blot of mouse tissue lysates

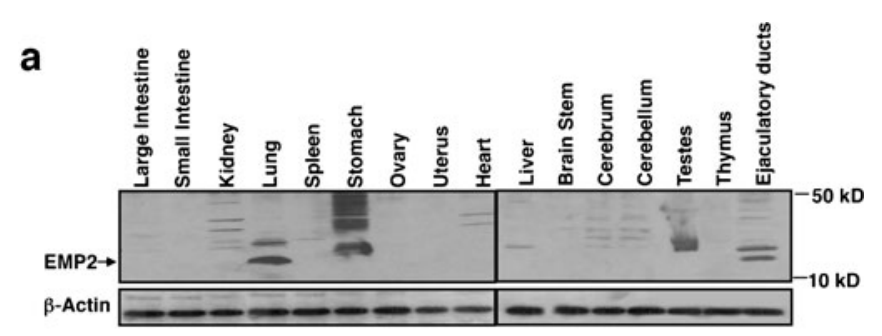

b

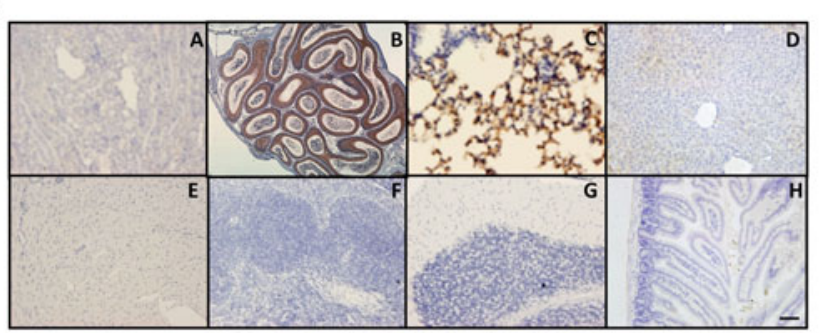

C

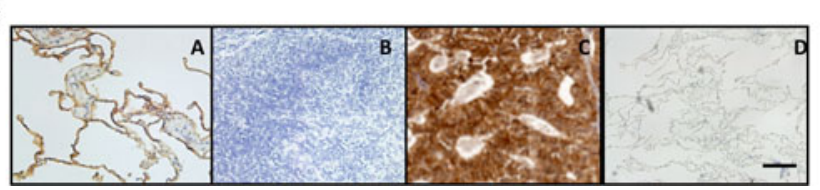

Fig. 1. Biodistribution and expression of the EMP2 protein. a Mouse tissue lysates were probed for EMP2 expression. The ejaculatory ducts include regions from the epididymis and vas deferens. Arrow depicts deglycosylated EMP2 (18 kD). b Immunohistochemistry analysis of mouse organs. The organs are designated as follows: $A$ kidney, $B$ epididymis, $C$ lung, $D$ liver, $E$ heart, $F$ spleen, $G$ brain, $H$ jejunum. c Immunohistochemistry analysis of human organs. The representative organs are designated as follows: $A$ lung, $B$ spleen, $C$ endometrial tumor, $D$ isotype control in the lung. 
revealed high expression of EMP2 in the lung, and some expression in vas deferens (Fig. 1a). Immunohistochemistry confirmed EMP2 expression in the epididymis and lung (panels $\mathrm{B}$ and $\mathrm{C}$ ) and no expression in the kidney, liver, heart, spleen, brain, and jejunum (Fig. 1b, panels A, D, E, F, $\mathrm{G}, \mathrm{H}$, respectively). As there is a $76 \%$ amino acid homology between human and mouse EMP2 (GeneBank numbers AAC51779.1 and AAK29076.1), we next evaluated the expression of EMP2 in a human tissue array using antiEMP2 antisera [22]. Similar to previous reports, EMP2 was highly expressed in alveolar epithelium of the lung [28, 29], in the retinal pigmented epithelium in the eye [22], and within secretory endometrium [23, 30], as well as in the epithelia of the vagina and fallopian tube (Table 1). In contrast, detailed immunohistochemical analysis revealed that EMP2 was undetectable in many human tissues including the small and large intestines, pancreas, liver, spleen, and kidney. To confirm its expression in humans, additional full section tissues were stained. EMP2 expression was readily detectable in the lung (Fig. 1c, panel A) but not in the spleen (panel B). In addition, high levels of EMP2 are seen in representative example of endometrial adenocarcinoma (panel C). The protein expression of EMP2 in these tissues appeared to be specific as no staining was observed using preimmune sera (lung, panel D). The limited normal tissue distribution of human EMP2 makes it an attractive target and suggests its suitability as a molecular target for immunoPET.

\section{Characterization of Purified KS83 Minibody}

Previous experience from our group has shown that the optimum antibody fragment for imaging is an intermediate sized antibody, termed a minibody (Fig. 2a). Minibodies show prolonged serum residence $\left(T_{1 / 2 \beta}=6 \mathrm{~h}\right)$ compared with a diabody $\left(T_{1 / 2 \beta}=2-4 \mathrm{~h}\right)$ [31] and have been used successfully in clinical trials to detect tumors prior to surgical excision [32]. The KS83 diabody was modified to create a minibody, and in accord with expectations, purified KS83 minibody formed a covalent homodimer with an estimated molecular mass of $75 \mathrm{kDa}$ under non-reducing conditions (Fig. 2b). Under reducing conditions, the KS83 minibody was dissociated into a monomer of approximately $40 \mathrm{kDa}$.

Table 1. Summary of EMP2 staining in a normal human tissue array

\begin{tabular}{llll}
\hline EMP2-positive tissue & \multicolumn{2}{l}{ EMP2-negative tissue } & \\
\hline Lung & Parathyroid & Thyroid & Testes \\
Skin & Adipose & Liver & Skeletal muscle \\
Fallopian tube & Esophagus & Stomach & Small intestine \\
Placenta & Colon & Anal mucosa & Gall bladder \\
Vagina & Omentum & Adrenal gland & Pancreas \\
Uterus & Spleen & Thymus & Parotid gland \\
& Heart & Kidney & Bladder \\
& Submandibular gland & Reactive lymph node \\
\hline
\end{tabular}

Size-exclusion chromatography also showed the formation of a dimer with a protein retention time at $31.36 \mathrm{~min}$ (average of two experiments), matching with the expected size of the minibody (Fig. 2c [20]). The ability of the KS83 minibody to bind to EMP2 was assessed directly on the HEC1A/EMP2 by flow cytometry. The KS 83 minibody demonstrated clear binding to the surface of HEC1A/EMP2 cells, and its affinity was similar to that observed for KS83 diabody (Fig. 2d). In contrast, the isotype control anti-CD20 minibody exhibited no binding to the EMP2-positive cells. To confirm that the KS83 minibody recognized murine EMP2, D2F2 mammary tumor cells were utilized. D2F2 cells have moderate surface expression of EMP2 (Fu and Wadehra, unpublished data), which was detected by the KS83 minibody (Fig. 2e). This effect seemed to be specific as the anti-CD20 minibody showed no binding to D2f2 cells. Furthermore, as shown in Fig. 2f, Ramos cells expressed high levels of CD20, but no EMP2 expression was found on the cells. These results indicate that the KS83 minibody specifically binds to EMP2 and recognizes native EMP2 expressed on the surface of both murine and human cells.

\section{EMP2 Internalizes Rapidly in the Presence of Anti-EMP2 Fragments}

Antibody binding to its receptor often leads to receptor internalization [33, 34]. To determine if EMP2 internalizes upon binding of an anti-EMP2 antibody fragment, light microscopy of HEC1A/EMP2 cells incubated with the antiEMP2 KS83 diabody was performed using a fluoresceinconjugated anti-Myc antibody. As seen in Fig. 3a, binding of KS83 diabody to HEC1A/EMP2 cells occurred within $10 \mathrm{~min}$ and remained cell-associated for up to $1 \mathrm{~h}$, but was lost by $24 \mathrm{~h}$. When the EMP2 levels in response to treatment with the anti-EMP2 antibody fragment was monitored, the EMP2 levels on the HEC1A/EMP2 cell surface were notably reduced within $24 \mathrm{~h}$ relative to the negative control cells treated with the A10 isotype control diabody (Fig. 3b). These results suggested that binding of anti-EMP2 antibody fragments leads to receptor internalization and degradation of the antibody fragment.

\section{DOTA-Conjugated KS83 Minibody and Binding Analysis of Bioconjugates}

Given the rapid internalization of antibody-bound EMP2, a residualizing radiolabel strategy was employed to visualize tumors in vivo. Residualizing radiolabels, such as radioactive metals attached to a bifunctional chelator such as DOTA, become trapped in the cells and are therefore retained within the cell longer than non-residualizing radiolabels [35]. The KS83 minibody was conjugated to DOTA and binding of the immunoconjugate to EMP2 was evaluated by flow cytometry (Fig. 4a, b). The conjugation conditions were investigated at different molar ratios of DOTA to KS 83 
a

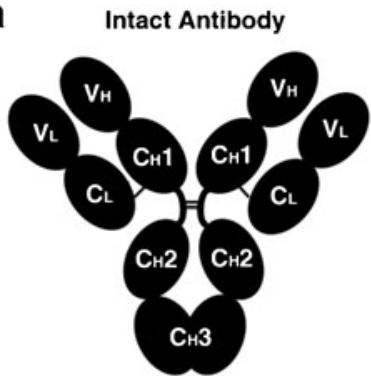

Diabody Minibody

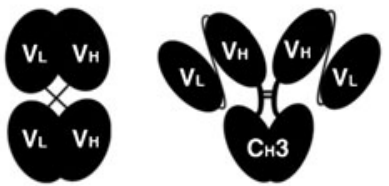

b

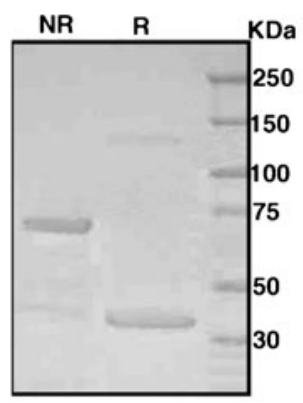

d

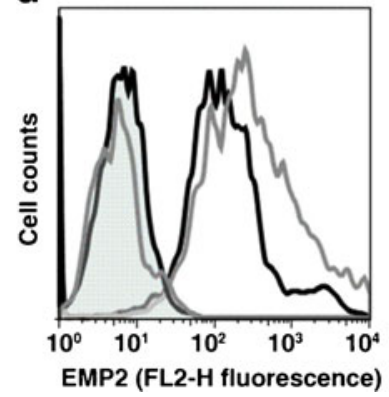

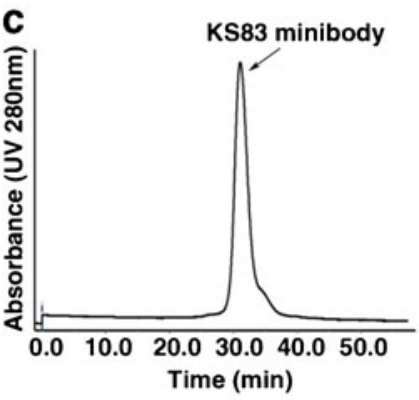

e

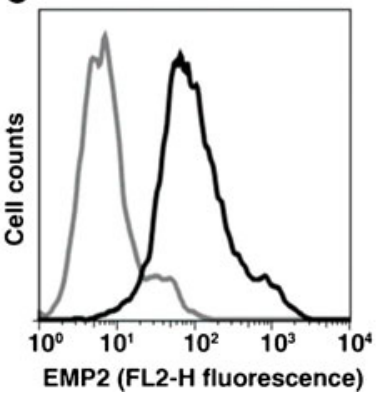

f

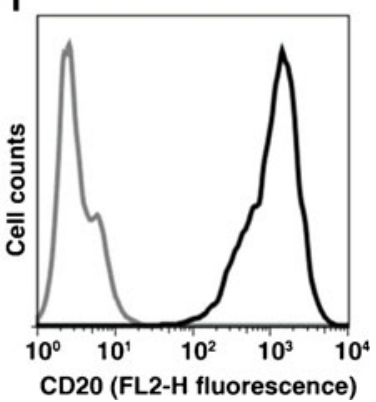

Fig. 2. Characterization of purified KS83 minibody. a Schematic representation of an intact antibody (150 kDa) and engineered antibody fragments including diabody (scFv dimer, $\sim 55 \mathrm{kDa}$ ) and minibody (scFv-hinge- $\mathrm{C}_{\mathrm{H}} 3$ dimer, $\sim 80 \mathrm{kDa}$ ). b Coomassie blue staining after SDS-PAGE of purified KS83 minibody under non-reducing (NR) and reducing conditions (R). Lane 3 Molecular weight marker. c Size-exclusion chromatography of purified KS83 minibody on a Superdex 200 column. Retention time of the sample was compared with appropriate molecular weight standards. $\mathbf{d}$ Flow cytometric analysis of HEC1A/EMP2 cells stained with antibody fragments KS83 minibody (black line), KS83 diabody (gray line), or isotype control anti-CD20 minibody (tinted black line) and A10 diabody (tinted gray line) and detected by phycoerythrin-conjugated goat antihuman Fc-specific antibody. e Flow cytometric analysis of murine D2F2 cells stained with KS83 minibody (black line). Anti-CD20 minibody was used as the isotype control (gray line) and detected as above. $\mathbf{f}$ Flow cytometric analysis of Ramos cells stained with anti-CD20 minibody (black line). KS83 minibody was used as the isotype control (gray line) and detected as above.

minibody in order to maximally preserve the binding to EMP2 (Fig. 4a). The optimal ratio of DOTA to KS83 minibody was found to be 20:1, and the number of DOTA per minibody $(\sim 3)$ was estimated based on the 20 lysines residues present in the KS83 minibody. At this conjugation ratio, binding activity was similar to that of the unconjugated minibody (Fig. 4b). The EMP2-binding of the DOTAconjugated KS83 minibody was verified by ELISA using EMP2 peptide-coated plates (Fig. 4c). Serial dilutions of unconjugated KS83 and DOTA-conjugated KS83 minibody revealed similar $\mathrm{EC}_{50}$ of $24 \mathrm{ng} / \mathrm{ml}(0.30 \mathrm{nM})$ and $29 \mathrm{ng} / \mathrm{ml}$ $(0.36 \mathrm{nM})$, respectively. Hence, the 20:1 DOTA to minibody ratio was used in all subsequent conjugation reactions.

\section{Small-Animal PET Imaging using Anti-EMP2 ${ }^{64}$ Cu-DOTA-Conjugated KS83 Minibody}

The DOTA-KS83 minibody was radiolabeled with ${ }^{64} \mathrm{Cu}$, and tumor targeting was evaluated in mice bearing HEC1A/ EMP2, HEC1A, and Ramos (EMP2-negative) xenografts. Although both wild-type HEC1A and HEC1A/EMP2 cells were injected on the same day, the growth rates of these cells differ in vivo [8]. The average weight of HEC1a/EMP2 xenografts was $158 \pm 50$ while HEC1A wild-type tumors were significantly smaller $(26 \pm 11 \mathrm{mg})$. As a negative control, Ramos cells were injected and imaged when they reached $232 \pm 23 \mathrm{mg}$. The radiolabeling efficiency for 
a

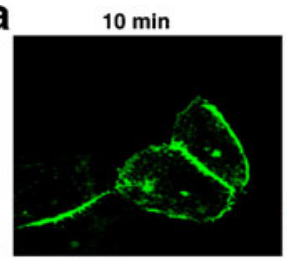

b

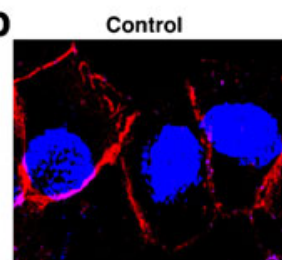

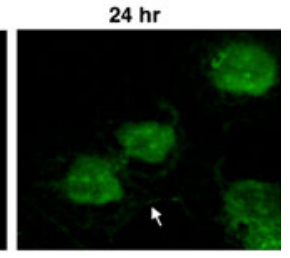

24 hrs (KS83)

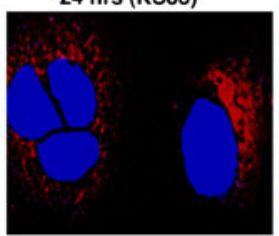

$24 \mathrm{hr}(\mathrm{A10})$

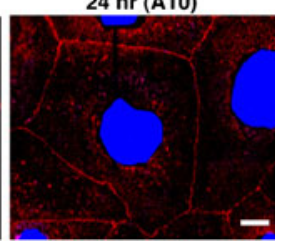

Fig. 3. EMP2 internalizes in the presence of anti-EMP2 fragments. a Localization of anti-EMP2 antibody (KS83 diabody) fragment (using fluorescein-conjugated anti-Myc antibody) on HEC1A/EMP2 after incubation for $10 \mathrm{~min}, 1 \mathrm{~h}$, and 24 h. b EMP2 localization detected by polyclonal antiEMP2 antibody (red) shows internalization in response $24 \mathrm{~h}$ after incubation with anti-EMP2 (KS83 diabody). Isotype control (A10 diabody) antibody fragments or no treatment (control) shows no internalization of EMP2. 4',6-Diamidino-2phenylindole was used for nuclear stain (b/ue).

DOTA-KS83 minibody was $97 \%$, and the immunoreactivity, or the biologically active fraction is the 64Cu-DOTAKS83 minibody that still retains binding to the target, was $84 \%$. Decay-corrected coronal micro-PET images were obtained at 4 and $20 \mathrm{~h}$ after intravenous injection with ${ }^{64} \mathrm{Cu}$ DOTA-KS83 minibody (Fig. 5a).

At both time points, HEC1A/EMP2 and wild-type tumors were clearly visualized, and a representative image at $20 \mathrm{~h}$ showed strong uptake in HEC1A/EMP2 tumor (white ellipse). As the HEC1A tumor is small, minimal uptake was visible in this coronal layer, but uptake was observed within the transverse profile (yellow ellipse) (Fig. 5a). In contrast, the EMP2-negative Ramos tumor had low minibody uptake (Fig. 5b). Radioactive uptake was also observed in the lung, heart, liver, and kidney at $4 \mathrm{~h}$ post-injection, with high activity persisting in the liver and kidney at $20 \mathrm{~h}$ post-injection.

Levels of radioactive uptake $(\% \mathrm{ID} / \mathrm{g})$ at 4 and $20 \mathrm{~h}$ were quantitated by both ROI analysis and biodistribution studies (Table 2). At $4 \mathrm{~h}$, ROI analysis showed significantly higher radioactive uptake using the ${ }^{64} \mathrm{Cu}$-DOTA-KS83 minibody in HEC1A/EMP2 tumors $(7.6 \pm 1.2 \% \mathrm{ID} / \mathrm{g})$ relative to $\mathrm{HEC} 1 \mathrm{~A}$ $(5.6 \pm 0.7 \% \mathrm{ID} / \mathrm{g} ; P<0.05)$ and Ramos tumors $(2.6 \pm 1.0 \% \mathrm{ID} / \mathrm{g}$; $P<0.001)$. HEC1A tumors also displayed significantly more radioactive uptake compared with that of Ramos tumor $(P<$ $0.01)$. RO1 analysis also detailed elevated radioactive uptakes in the kidneys, liver, heart, and lungs, but low levels were observed within soft tissue. After 20 h, HEC1A/EMP2 tumors showed a 1.7 -fold increase in ${ }^{64} \mathrm{Cu}$-DOTA-KS83 minibody localization compared with the wild-type tumor $(p<0.01)$ and a 5.4-fold increase in localization compared with Ramos tumors $(P<0.001)$. The difference between HEC1A and Ramos
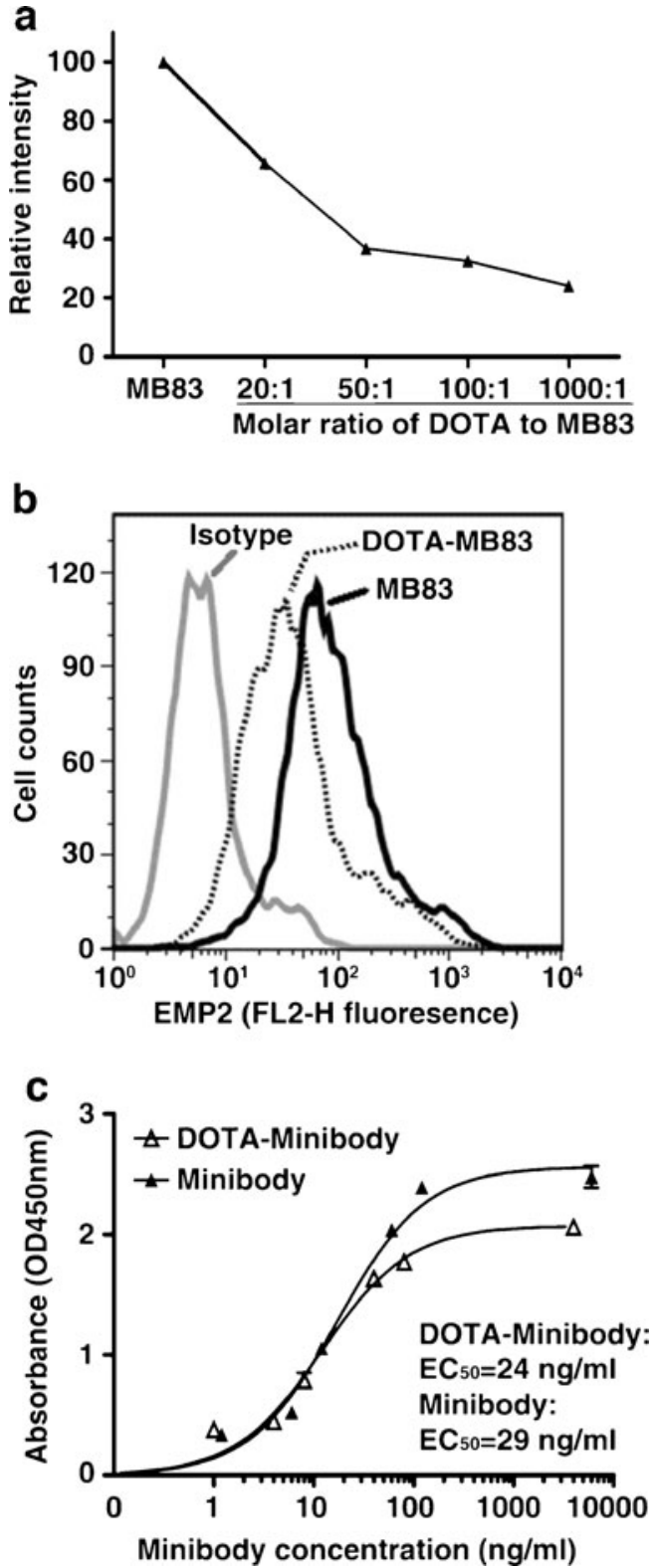

Fig. 4. Conjugation of KS83 minibody with DOTA and binding analysis of subsequent bioconjugates. a The effect of varying the molar ratios of DOTA to KS83 minibody was determined by flow cytometry using HEC1A/EMP2 cells. The optimal molar ratio of DOTA to KS83 is $20: 1$, as shown by the best preserved binding activity of the DOTA-KS83 bioconjugate to the cells. b Representative flow cytometric image of HEC1A/EMP2 cells stained with unconjugated KS83 minibody and the 20:1 conjugated DOTA-KS83 minibody. Unconjugated KS83 minibody and the isotype control A10 diabody are depicted as the positive and negative controls, respectively. c The level of binding of the KS83 and DOTA-KS83 minibody to human EMP2 second extracellular loop peptide was determined by ELISA. Plates were coated with the 24 amino acid human EMP2 peptide, and bound minibodies were detected with HRP-conjugated goat antihuman Fc-specific antibody. 
a

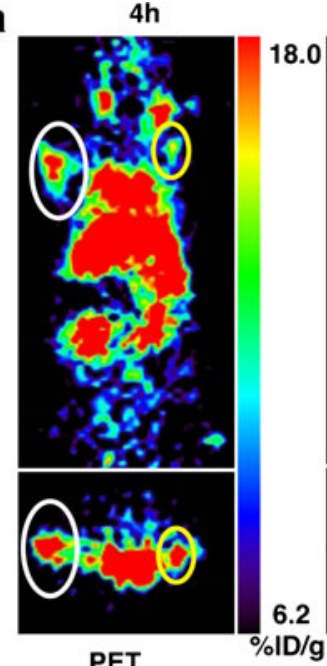

PET

b

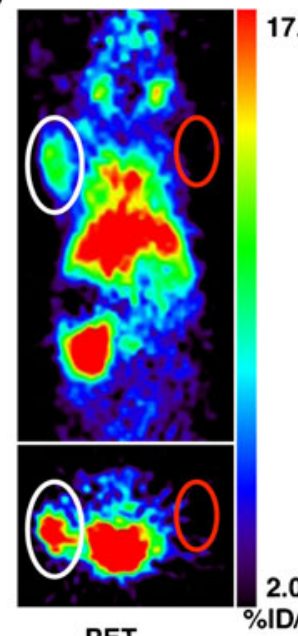

PET

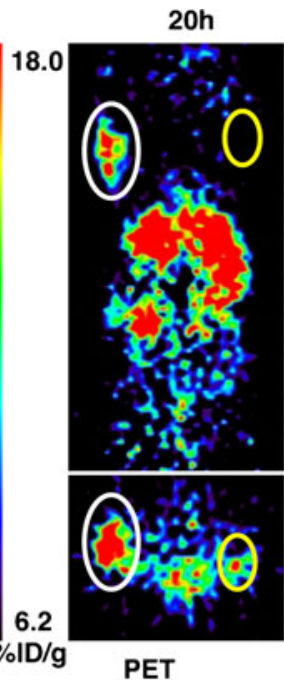

PET

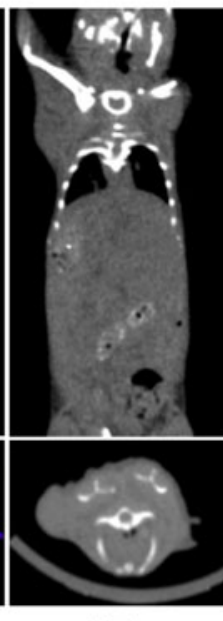

CT

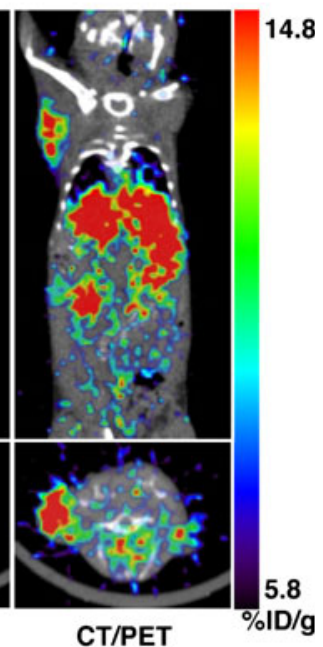

CT/PET
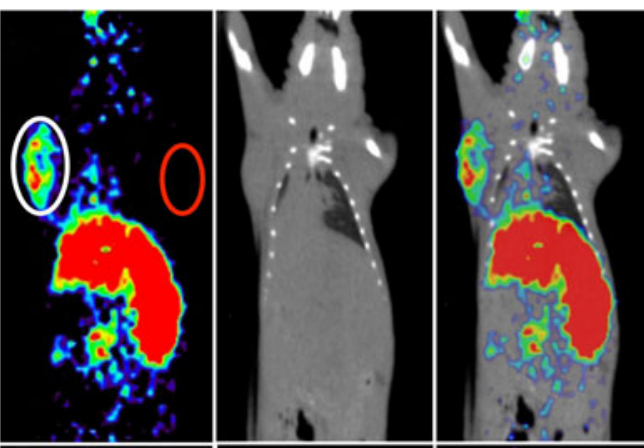

15.2

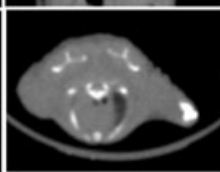

CT

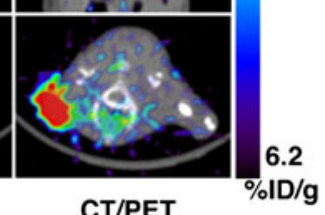

CT/PET

White ellipse: HEC1A/EMP2 tumor

Red ellipse : Ramos tumor

Yellow ellipse: HEC1A tumor

Fig. 5. Small-animal PET imaging of EMP2 using ${ }^{64} \mathrm{Cu}-\mathrm{DOTA}-\mathrm{KS} 83$ minibody. a Small-animal PET coronal images of mice bearing HEC1A/EMP2 (left shoulder, denoted white ellipse) and HEC1A (right shoulder, denoted yellow ellipse) tumor xenografts following administration of ${ }^{64} \mathrm{Cu}$-labeled anti-EMP2 DOTA-KS83 minibody at 4 and $20 \mathrm{~h}$. Bottom inset shows a transverse image from the same animal. b Small-animal PET coronal images of mice bearing xenografts from HEC1A/EMP2 (left shoulder, denoted white ellipse) and Ramos (right shoulder, denoted red ellipse) cells. PET, CT, and PET/CT overlay images are shown at $20 \mathrm{~h}$ time point. Bottom inset depicts a transverse image from the same animal. Images at both time points are representative of five or more mice.

tumors was also significant as wild-type tumors showed a 3.9-fold increase in radiolabeled KS83 localization $(P<$ $0.05)$. In order to validate the ROI uptakes, tumors and the major organs were excised from mice at either 4 or $20 \mathrm{~h}$ post-injection, and the activity in the organs was counted in order to determine the percent injected dose per gram (Table 2). The counts from ROI analysis and ex vivo biodistribution were similar except that a slight difference was observed in the kidney.

The data generated suggest that anti-EMP2 minibodies localize specifically and with sensitivity to EMP2 positive tumors. However, an alternative possibility may be that EMP2 positive tumors differ in their vascularity and/or preferentially retain the radiometal labeled minibody. To explore the second possibility, experiments were performed using a ${ }^{64} \mathrm{Cu}$-DOTA CD20 minibody on HEC1A/EMP2 tumors. Table 3 compares the biodistribution of the control isotype tracer with the KS83 minibody at time of killing (4 and $20 \mathrm{~h}$ ). Anti-EMP2 minibodies showed a 1.2- and 3-fold increase in uptake compared with radiolabeled anti-CD20 at 4 and $20 \mathrm{~h}$, respectively. 
Table 2. Estimated activities from drawn ROIs and uptakes measured by ex vivo weighing and counting derived from ${ }^{64} \mathrm{Cu}$-DOTA-KS83 $(n=5)$

\begin{tabular}{lrrrr}
\hline Organ $(\% \mathrm{ID} / \mathrm{g})$ & ROI $(4 \mathrm{~h})$ & ROI $(20 \mathrm{~h})$ & \multicolumn{1}{l}{ Bio $(4 \mathrm{~h})$} & Bio $(20 \mathrm{~h})$ \\
\hline $\begin{array}{l}\text { HEC1A/EMP2 } \\
\quad \text { tumor }\end{array}$ & $7.6 \pm 1.2$ & $10.2 \pm 2.6$ & $7.3 \pm 0.5$ & $9.7 \pm 1.9$ \\
Hec1A tumor & $5.6 \pm 0.7$ & $6.0 \pm 0.1$ & $5.8 \pm 0.8$ & $4.2 \pm 1.3$ \\
Ramos tumor & $2.6 \pm 1.0$ & $1.9 \pm 0.5$ & N.D. & $2.3 \pm 0.5$ \\
Liver & $19.6 \pm 1.9$ & $19.6 \pm 3.5$ & $19.2 \pm 5.4$ & $18.1 \pm 1.6$ \\
Kidney & $26.8 \pm 4.6$ & $24.8 \pm 5.9$ & $47.1 \pm 12.4$ & $36.4 \pm 8.3$ \\
Lung & $9.7 \pm 1.6$ & $4.8 \pm 1.2$ & $12.5 \pm 5.6$ & $5.5 \pm 1.1$ \\
Heart & $13.9 \pm 2.8$ & $4.2 \pm 0.4$ & $9.2 \pm 0.9$ & $4.3 \pm 1.0$ \\
Soft tissue & $0.7 \pm 0.2$ & $0.7 \pm 0.2$ & $1.0 \pm 0.4$ & $0.8 \pm 0.1$ \\
Blood & N.D. & N.D. & $18.1 \pm 3.0$ & $3.7 \pm 0.6$ \\
Uterus & N.D. & N.D. & $5.4 \pm 0.8$ & $4.9 \pm 1.6$ \\
\hline
\end{tabular}

Data are expressed as $\% \mathrm{ID} / \mathrm{g} \pm \mathrm{SD}$

N.D. not determined

\section{Discussion}

EMP2 is a tetraspan protein that is upregulated in a number of gynecological cancers, including endometrial and ovarian $[3$, 13]. Using a large panel of mouse and human biospecimens, we observed a restricted distribution of EMP2 protein expression in normal tissues in both species, suggesting the utility of EMP2 as a therapeutic and pharmacodiagnostic target. While EMP2 expression in human tissues has been previously studied, this has largely been evaluated at the level of messenger RNA (mRNA) rather than protein expression; however, the latter is most relevant to imaging strategies. In the present study, we show that EMP2 protein expression is largely concordant with mRNA expression, with EMP2 highly expressed in alveolar epithelium of the lung [28, 29], in the retinal pigmented epithelium in the eye [22], within secretory endometrium $[23,30]$, and in epithelia of the vagina and fallopian tube. In contrast, EMP2 was undetectable in many human tissues, including the small and large intestines, pancreas, liver, spleen, anovulatory endometrium, and kidney.

There is an unmet need for novel imaging strategies for clinical management of endometrial and ovarian cancers. In this paper, we show as a proof of principle that EMP2 expression can serve as a target for imaging. We have previously shown that the KS83 diabody recognizes both murine and human EMP2 and has therapeutic potential as seen in a number of tumor models [3, 13]. We thus hypothesized that imaging of EMP2 may serve as an early

Table 3. Biodistribution (bio) uptake of a control isotype tracer ${ }^{64} \mathrm{Cu}$ DOTA-CD20 at time of killing $(n=2)$

\begin{tabular}{lrr}
\hline Organ $(\% \mathrm{ID} / \mathrm{g})$ & \multicolumn{1}{c}{$4 \mathrm{~h}$} & \multicolumn{1}{c}{$20 \mathrm{~h}$} \\
\hline Hec1A/EMP2 tumor & $6.1 \pm 0.1$ & $3.4 \pm 0.6$ \\
Liver & $19.0 \pm 3.1$ & $26.6 \pm 0.9$ \\
Kidney & $33.1 \pm 8.6$ & $24.2 \pm 8.2$ \\
Lung & $12.1 \pm 2.6$ & $6.2 \pm 0.9$ \\
Heart & $8.3 \pm 2.1$ & $6.2 \pm 0.7$ \\
Soft tissue & $1.1 \pm 0.8$ & $0.8 \pm 0.3$ \\
Blood & $13.2 \pm 1.6$ & $3.1 \pm 1.9$ \\
Uterus & $5.6 \pm 2.7$ & $4.9 \pm 0.6$ \\
\hline
\end{tabular}

Mean is measured with SD monitor of response to tailored therapy or be useful prior to surgical resection to identify metastatic disease. Therefore, we reformatted the variable genes of the KS83 diabody [13] into a minibody of approximately $80 \mathrm{kDa}$ in size whose pharmacokinetics and biodistribution are particularly favorable compared to other antibody fragments for tumor imaging [21]. Minibodies have a slower clearance compared to the diabody largely due to their increased molecular weight, and they have been shown to be useful for PET imaging [20].

Preliminary experiments showed that antibody fragment binding to EMP2 caused the target to internalize. Indeed, initial imaging studies using nonresidualizing anti-EMP2 antibody fragments (prepared with I-124 by the Iodogen method) produced poor retention in the EMP2-positive tumor xenografts (data not shown). This was not unexpected as antigen internalization releases radioiodine by dehalogenases either directly or after the protein is degraded to iodotyrosines, resulting in loss of signal and low percent injected dose per gram [36].

Accordingly, in this study, we prepared a residualizing anti-EMP2 radiolabel using the bifunctional chelating agent DOTA to conjugate minibody with a chelator for ${ }^{64} \mathrm{Cu}[24$, 37-39]. ${ }^{64} \mathrm{Cu}$-DOTA has been shown to have moderate stability in vivo, which leads to a high hepatic radioactivity uptake [40]. ${ }^{64} \mathrm{Cu}$-radiolabeled complexes with increased stability have been studied with 1,4,8,11-tetraazacyclotetradecane- $N, N^{\prime}, N^{\prime \prime}, N^{\prime \prime \prime}$-tetraacetic acid $[40,41]$, but this chelator requires harsh reaction conditions such as elevated temperature for complexation with the radiometal. Although the 1,4,7-triazacyclononane- $N, N^{\prime}, N^{\prime \prime}$-triacetic acid can be labeled in mild conditions and provides better stability of Cu-chelate complexes $[42,43]$, it has not been evaluated in the clinic. Thus, in this study, we used DOTA-mono- $N$ hydroxysuccinimide ester (DOTA-NHS-ester) conjugated with lysine residues of KS83 minibody in order to radiometal label with ${ }^{64} \mathrm{Cu}$. Since lysine residues are present in loops forming the antigen binding site, modification of the lysine residues could potentially affect binding to the antigen. Indeed, molar ratios of 50:1 and over greatly impacted binding to EMP2 (Fig. 4a). However, a molar ratio of 20:1 largely preserved the antigen binding activity compared to unmodified KS83 minibody. Hence, this conjugation strategy proved to be suitable for radiolabeling the anti-EMP2 KS83 minibody.

Non-invasive serial PET imaging was used to generate data in the same animal, providing important information of how the compound moves through the body over time. Indeed, PET images of tumor bearing mice injected with ${ }^{64} \mathrm{Cu}$-DOTA-KS83 minibody produced excellent radioactive uptake in EMP2positive tumors. At $20 \mathrm{~h}$, which is the optimal time for minibody imaging [37], drawn ROIs revealed $10.2 \pm 2.6 \% \mathrm{ID} / \mathrm{g} \pm \mathrm{SD}$ in the HEC1A/EMP2 tumor, a 5.4-fold uptake ratio compared to that in the EMP2-negative tumor $(1.9 \pm 0.5)$. These findings were confirmed directly ex vivo by measuring the radioactivity in excised organs. 
In order to determine the sensitivity of ${ }^{64} \mathrm{Cu}$-DOTAKS83 minibody to EMP2, xenografts were created using both wild-type HEC1A and HEC1A/EMP2 cells. Published reports have shown that there is a 2- to 4-fold increase in EMP2 expression by Western blot analysis [8]. Imaging with the ${ }^{64} \mathrm{Cu}$-DOTA-KS83 minibody demonstrated targeting to both HEC1A/EMP2 $(10.2 \pm 2.6 \% \mathrm{ID} / \mathrm{g})$ and HEC1A $(6.0 \pm 0.1 \% \mathrm{ID} / \mathrm{g})$ tumors, with HEC1A/EMP2 exhibiting a 1.7-fold higher level of radioactivity compared to HEC1A $(P<0.01)$. Moreover, the high ratio of EMP2-positive tumor relative to various normal tissues lacking EMP2 protein expression (e.g., soft tissue, heart, blood) suggests that EMP2 minibodies specifically and efficiently adhere to their antigen. To verify that the retention was not due to permeability and retention effects, HEC1A/EMP2 xenografts were targeted using a control isotype tracer. Injections with radiometal-labeled anti-CD20 minibody showed low levels of tracer within the tumor, suggesting that the high radiouptake of labeled EMP2 minibody was specific.

Of note, high radioactive uptake of ${ }^{64} \mathrm{Cu}$-DOTA-KS83 minibody was observed in liver and kidney even though these are not sites of EMP2 expression. This uptake may be attributed in part to the minibody or the radiometal itself. The liver uptake was expected, due to the size of the minibody that is above the renal threshold for clearance $(>60 \mathrm{kDa})$ and possibly by avid transchelation of the radiometal ${ }^{64} \mathrm{Cu}$ [40]. The elevated renal uptake was unexpected, but may reflect the small fraction of partially metabolized ${ }^{64} \mathrm{Cu}$-DOTA-KS83 minibody, which despite residualizing properties are released into circulation from primary tissue binding sites and reach the kidney where the partially metabolized products may be retained. This may also explain the slightly elevated levels of agent detectable at $20 \mathrm{~h}$ in the blood and heart (the latter largely reflecting a blood compartment signal). Consistent with this explanation, high radioactive uptake was also observed using the ${ }^{64} \mathrm{Cu}$ DOTA-CD20 minibody.

The present and previous studies show that EMP2 is highly expressed in normal lung (type 1 pneumocytes) [44]. As the minibody recognizes both human and murine EMP2, it is notable that lung uptake of ${ }^{64} \mathrm{Cu}$-DOTA-KS83 minibody is $<2$-fold higher than the activity in the blood. This suggests that EMP2 in native pulmonary epithelium may be relatively sequestered from access to ${ }^{64} \mathrm{Cu}-\mathrm{DOTA}-\mathrm{KS} 83$ minibody. Consistent with this hypothesis, localization of EMP2 in polarized epithelia is restricted to zona adherens (Morales and Gordon, unpublished data). Nonetheless, the expression of EMP2 may make it difficult to detect and characterize lung metastases. Additional experiments will be needed to verify these and other utilities.

In conclusion, EMP2 is novel cancer biomarker, notably for endometrial and ovarian cancer, that has been previously shown to be an independent prognostic indicator, and an effective target in preclinical recombinant antibody therapy. In this study, we characterize a residualizing imaging agent, a ${ }^{64} \mathrm{Cu}$-labeled minibody to EMP2, and demonstrate its effectiveness in imaging human EMP2-positive endometrial tumor xenografts. We predict that with further refinement anti-EMP2 antibody fragments may be have value for imaging of tumor localization and therapeutic response in patients with EMP2-positive malignancies.

Acknowledgments. This work was supported by NIH grants HD48540 (J. Braun), R21 CA131756 (M. Wadehra), CA016042 (University of California at Los Angeles Jonsson Comprehensive Cancer Center flow cytometry core), and R24 CA86307 (Production of ${ }^{64} \mathrm{Cu}$ at Washington University School of Medicine). A.M.W., J.S., J.B., and M.W. are members of the Jonsson Comprehensive Cancer Center. We are especially grateful to Dr. David Ho and Arye Lipman in ImaginAb, Inc. for the assistance with minibody KS83 construction and Dr. David Stout and Waldemar Ladno at the Crump Institute for Molecular Imaging at UCLA (U24 CA092865) for the assistance with the small animal PET/CT scans.

Conflict of Interest. M.W., L.K.G, and J.B. have patent rights for the EMP2 minibody.

Open Access. This article is distributed under the terms of the Creative Commons Attribution License which permits any use, distribution, and reproduction in any medium, provided the original author(s) and the source are credited.

\section{References}

1. Wadehra M, Natarajan S, Seligson DB et al (2006) Expression of epithelial membrane protein-2 is associated with endometrial adenocarcinoma of unfavorable outcome. Cancer 107:90-98

2. Habeeb O, Goodglick L, Soslow RA et al (2010) Epithelial membrane protein-2 expression is an early predictor of endometrial cancer development. Cancer 116:4718-4726

3. Fu M, Maresh EL, Soslow RA et al (2010) Epithelial membrane protein-2 is a novel therapeutic target in ovarian cancer. Clin Cancer Res 16:3954-3963

4. Wadehra M, Goodglick L, Braun J (2004) The tetraspan protein EMP2 modulates the surface expression of caveolins and glycosylphosphatidyl inositol-linked proteins. Mol Biol Cell 15:2073-2083

5. Forbes A, Wadehra M, Mareninov S et al (2007) The tetraspan protein EMP2 regulates expression of caveolin-1. J Biol Chem 282:2654226551

6. Morales SA, Mareninov S, Wadehra M et al (2009) FAK activation and the role of epithelial membrane protein 2 (EMP2) in collagen gel contraction. Invest Ophthalmol Vis Sci 50:462-469

7. Wadehra M, Forbes A, Pushkarna N et al (2005) Epithelial membrane protein-2 regulates surface expression of alphavbeta3 integrin in the endometrium. Dev Biol 287:336-345

8. Fu M, Rao R, Sudhakar D et al (2011) Epithelial membrane protein-2 promotes endometrial tumor formation through activation of FAK and Src. PLoS One 6:e19945

9. Berditchevski F (2001) Complexes of tetraspanins with integrins: more than meets the eye. J Cell Sci 114:4143-4151

10. Levy S, Shoham T (2005) The tetraspanin web modulates immunesignalling complexes. Nat Rev Immunol 5:136-148

11. Maecker HT, Todd SC, Levy S (1997) The tetraspanin superfamily: molecular facilitators. FASEB J 11:428-442

12. Zevian S, Winterwood NE, Stipp CS (2011) Structure-function analysis of tetraspanin CD151 reveals distinct requirements for tumor cell behaviors mediated by alpha3beta1 versus alpha6beta4 integrin. J Biol Chem 286:7496-7506

13. Shimazaki K, Lepin EJ, Wei B et al (2008) Diabodies targeting epithelial membrane protein 2 reduce tumorigenicity of human endometrial cancer cell lines. Clin Cancer Res 14:7367-7377

14. Czernin J, Phelps ME (2002) Positron emission tomography scanning: current and future applications. Annu Rev Med 53:89-112

15. Gambhir SS (2002) Molecular imaging of cancer with positron emission tomography. Nat Rev Cancer 2:683-693

16. Stahl A, Wieder H, Piert M, Wester HJ, Senekowitsch-Schmidtke R, Schwaiger M (2004) Positron emission tomography as a tool for translational research in oncology. Mol Imaging Biol 6:214-224 
17. Dumont RA, Hildebrandt I, Su H et al (2009) Noninvasive imaging of alphaVbeta3 function as a predictor of the antimigratory and antiproliferative effects of dasatinib. Cancer Res 69:3173-3179

18. Lai CH, Yen TC, Chang TC (2007) Positron emission tomography imaging for gynecologic malignancy. Curr Opin Obstet Gynecol 19:37-41

19. Schoder H, Larson SM (2004) Positron emission tomography for prostate, bladder, and renal cancer. Semin Nucl Med 34:274-292

20. Olafsen T, Kenanova VE, Wu AM (2010) Generation of single-chain $\mathrm{Fv}$ fragments and multivalent derivatives $\mathrm{scFv}-\mathrm{Fc}$ and $\mathrm{scFv}-\mathrm{CH} 3$ (minibodies). In: Kontermann R, Dübel S (eds) Antibody engineering, vol 2. Springer, Berlin, pp 69-84

21. Olafsen T, Kenanova VE, Wu AM (2006) Tunable pharmacokinetics: modifying the in vivo half-life of antibodies by directed mutagenesis of the Fc fragment. Nat Protoc 1:2048-2060

22. Wadehra M, Sulur GG, Braun J, Gordon LK, Goodglick L (2003) Epithelial membrane protein-2 is expressed in discrete anatomical regions of the eye. Exp Mol Pathol 74:106-112

23. Wadehra M, Dayal M, Mainigi M et al (2006) Knockdown of the tetraspan protein epithelial membrane protein-2 inhibits implantation in the mouse. Dev Biol 292:430-441

24. Lewis MR, Kao JY, Anderson AL, Shively JE, Raubitschek A (2001) An improved method for conjugating monoclonal antibodies with Nhydroxysulfosuccinimidyl DOTA. Bioconjug Chem 12:320-324

25. Olafsen T, Betting D, Kenanova VE et al (2009) Recombinant antiCD20 antibody fragments for small-animal PET imaging of B-cell lymphomas. J Nucl Med 50:1500-1508

26. Defrise M, Kinahan PE, Townsend DW, Michel C, Sibomana M, Newport DF (1997) Exact and approximate rebinning algorithms for $3-$ D PET data. IEEE Trans Med Imaging 16:145-158

27. Loening AM, Gambhir SS (2003) AMIDE: a free software tool for multimodality medical image analysis. Mol Imaging 2:131-137

28. Ben-Porath I, Kozak CA, Benvenisty N (1998) Chromosomal mapping of Tmp (Emp1), Xmp (Emp2), and Ymp (Emp3), genes encoding membrane proteins related to Pmp22. Genomics 49:443-447

29. Wang CX, Wadehra M, Fisk BC, Goodglick L, Braun J (2001) Epithelial membrane protein 2, a 4-transmembrane protein that suppresses B-cell lymphoma tumorigenicity. Blood 97:3890-3895

30. Wadehra M, Mainigi M, Morales SA et al (2008) Steroid hormone regulation of EMP2 expression and localization in the endometrium. Reprod Biol Endocrinol 6:15

31. McCabe KE, Wu AM (2010) Positive progress in immunoPET-not just a coincidence. Cancer Biother Radiopharm 25:253-261

32. Wong JY, Chu DZ, Williams LE et al (2004) Pilot trial evaluating an 123I-labeled 80-kilodalton engineered anticarcinoembryonic antigen antibody fragment (cT84.66 minibody) in patients with colorectal cancer. Clin Cancer Res 10:5014-5021

33. Sunada H, Magun BE, Mendelsohn J, MacLeod CL (1986) Monoclonal antibody against epidermal growth factor receptor is internalized without stimulating receptor phosphorylation. Proc Natl Acad Sci USA 83:3825-3829

34. Barat B, Kenanova VE, Olafsen T, Wu AM (2011) Evaluation of two internalizing carcinoembryonic antigen reporter genes for molecular imaging. Mol Imaging Biol 13:526-535

35. Shokeen M, Anderson CJ (2009) Molecular imaging of cancer with copper-64 radiopharmaceuticals and positron emission tomography (PET). Acc Chem Res 42:832-841

36. Nayak TK, Brechbiel MW (2009) Radioimmunoimaging with longer-lived positron-emitting radionuclides: potentials and challenges. Bioconjug Chem 20:825-841

37. Wu AM, Yazaki PJ, Tsai S et al (2000) High-resolution microPET imaging of carcinoembryonic antigen-positive xenografts by using a copper-64-labeled engineered antibody fragment. Proc Natl Acad Sci USA 97:8495-8500

38. Niu G, Li Z, Cao Q, Chen X (2009) Monitoring therapeutic response of human ovarian cancer to 17-DMAG by noninvasive PET imaging with (64)Cu-DOTA-trastuzumab. Eur J Nucl Med Mol Imaging 36:15101519

39. Elsasser-Beile U, Reischl G, Wiehr S et al (2009) PET imaging of prostate cancer xenografts with a highly specific antibody against the prostate-specific membrane antigen. J Nucl Med 50:606-611

40. Boswell CA, Sun X, Niu W et al (2004) Comparative in vivo stability of copper-64-labeled cross-bridged and conventional tetraazamacrocyclic complexes. J Med Chem 47:1465-1474

41. Sprague JE, Peng Y, Fiamengo AL et al (2007) Synthesis, characterization and in vivo studies of $\mathrm{Cu}(\mathrm{II})$-64-labeled cross-bridged tetraazamacrocycle-amide complexes as models of peptide conjugate imaging agents. J Med Chem 50:2527-2535

42. Prasanphanich AF, Nanda PK, Rold TL et al (2007) [64Cu-NOTA-8Aoc-BBN(7-14)NH2] targeting vector for positron-emission tomography imaging of gastrin-releasing peptide receptor-expressing tissues. Proc Natl Acad Sci USA 104:12462-12467

43. Prasanphanich $\mathrm{AF}$, Retzloff $\mathrm{L}$, Lane $\mathrm{SR}$ et al (2009) In vitro and in vivo analysis of [(64)Cu-NO2A-8-Aoc-BBN(7-14)NH(2)]: a site-directed radiopharmaceutical for positron-emission tomography imaging of T47D human breast cancer tumors. Nucl Med Biol 36:171-181

44. Dahlin K, Mager EM, Allen L et al (2004) Identification of genes differentially expressed in rat alveolar type I cells. Am J Respir Cell Mol Biol 31:309-316 